\title{
Selection of Hepatocyte-Like Cells from Mouse Differentiated Embryonic Stem Cells and Application in Therapeutic Liver Repopulation
}

\author{
Xiao-Geng Deng Rong-Lin Qiu Zhi-Xi Li Jie Zhang Jia-Jia Zhou Yao-Hao Wu \\ Le-Xiang Zeng Jing Tang \\ Department of Pediatric Surgery, The Memorial Hospital of Sun Yat-Sen University, Guangzhou
}

\section{Key Words}

Embryonic stem cells(ESCs) - Hepatocyte - Cholestatic sera - Intrahepatic transplantation • Therapeutic liver repopulation $\bullet$ Farnesoid $X$ receptor (FXR)

\begin{abstract}
Backgroud/Aim: Because of the oncogenic risk, it is important to gain the homogeneous and purified cells from differentiated ESCs before transplantation. Here, we aim to select hepatocyte-like cells from differentiated ESCs, and investigate their growth, differentiation and neoplastic formation after intrahepatic transplantation. Methods: Mouse ESCs were primarily induced by Dexamethesone, FGF-4 and HGF sequentially, then placed to a conditioning selection media consisting of $5 \%$ cholestatic sera and cultivated for 2 wks. After labeled by CFDA-SE, the selected cells were transplanted into mouse liver in therapeutic liver repopulation models. Results: In the early stage of screening cultivation, most cells were suffered from apoptosis or even death. 1w later, some hepatocyte-like colony-forming units were observed, then the selected cells could grow and tend to be more mature, as assessed by morphological and functional tests. After intrahepatic transplantation, the labeled cells could proliferate and expressed albumin. Moreover, teratoma didn't form over 3 months. Conclusion: Our conditioning selection media could not only effectively select hepatocytelike cells from differentiated ESCs, but further promote their growth and differentiation as well. After intrahepatic transplantation in therapeutic liver repopulation models, the selected cells could grow, differentiate and keep partial hepatic function. In particular, the transplantation was safe.

Copyright $\odot 2012$ S. Karger AG, Basel

Dr. Xiao-Geng Deng




\section{Introduction}

The treatment of troublesome or end-stage liver diseases(ESLD), such as hepatitis, liver cirrhosis, primary liver carcinoma, congenital biliary atresia, inherited enzyme deficiency diseases and acute liver failure, is always a global difficulty. Orthotopic liver transplantation (OLT) is a effective treatment for patients with ESLD, but the shortage of organ donors limits the number of liver transplantations [1]. Hepatocyte transplantation (HTx) provides a new way for it, and maybe an alternative substitute therapy for these liver diseases [2]. However, the anticipated clinical efficacy of HTx has not been proved yet, mainly because of the limitations of the source deficiency and the proliferation difficulty of the transplanted hepatocytes [3-4].

The establishment of human embryonic stem cells (ESCs) lines will undoubtedly give much hope to solve the source deficiency of transplantable cells [5]. The distinguishing feature of ESCs is their pluripotent ability to differentiate into a broad spectrum of derivatives of all 3 embryonic germ layers. This ability has drawn attention to ESCs as a novel source of cell populations for new therapeutic strategies such as cell transplantation and tissue engineering. Actually, abundant studies have reported the capacity of ESCs to differentiate into hepatocytes or hepatocyte-like cells by different ways [6-10], but the propensity to develop teratomas about the ESCs-derived hepatocytes and low efficiency are still the dominating problems. So getting the homogeneous and secure cells suitable for transplanting is still important.

In fact, researchers had adopted various methods to select and purify hepatocyte-like cells from differentiated cells [11-14]. For example, Yamada et al. found that indocyanine green(ICG)-staining was a useful marker to identify differentiated hepatocyte-like cells from EBs in vitro. Since ICG is eliminated exclusively by hepatocytes, so ICG -staining cells can be isolated from other kinds of cells [12]. Yin et al. used AFP as a marker. After knocking the GFP gene into the AFP locus of ESCs and differentiating the modified ESCs in vitro, they got a subpopulation of GFP (+) and AFP-expressing hepatic-like cells, thus hepatocyte-like cells were isolated [13]. However, the methods mentioned above are either complex or have a poor isolation efficiency. Therefore, it is essential to establish not only an appropriate culture system to induce ESCs to hepatocytes, but also an useful method for isolating hepatocyte-like cells from differentiated ESCs to eliminate the potential danger of forming aggressive ESCsderived teratomas in vivo.

Here, we primarily induced mouse ESCs to differentiate into hepatocyte-like cells by adding Dex, FGF-4 and HGF sequentially, in accordance with the research proceeding of liver developmental biology. Then, on foundation of succeeding to select hepatic stem cells from bone marrow stem cells with a conditioning selection media consisting of 5\% cholestatic sera [15], we further investigated the effects of this pathological microenvironmental culture system on selecting hepatocyte-like cells from differentiated ESCs. Finally, we transplanted these induced and selected cells into mice liver through portal vein, in therapeutic liver repopulation mice models which were pre-treated with retrorsine and $70 \%$ partial hepatotomy. We would confirm whether the transplanted cells could be normally incorporated into mice liver parenchymal structure and whether they could further proliferate, keep the hepatic function or form teratomas. Our research may lay a foundation for the clinical application of ESCs as a novel source of HTx.

\section{Materials and Methods}

Culture of ESCS

E14 mouse ESCs were maintained in feeder-free DMEM (Dulbecco's modified eagle medium) with high glucose (Gibco BRL, Rockville MD), $1000 \mathrm{U} / \mathrm{ml}$ recombinant mouse leukemia inhibitory factor (rmLIF) (Chemicon), 10\% fetal bovine serum FBS (Hyclone), $0.1 \mathrm{mmol} / \mathrm{L} \beta$-mercaptoethanol (Chemicon), $10 \mathrm{ml} / \mathrm{L}$ 
Insulin-Transferrin-sodium selenite (Gibco) and 1\% nonessential amino acids (Chemicon), $100 \mathrm{u} / \mathrm{ml}$ penicillin, $100 \mu \mathrm{g} / \mathrm{ml}$ streptomycin. The density of ESCs was approximately $1 \times 10^{6} / \mathrm{ml}$.

\section{Initial proliferation and differentiation of ESCS}

When mouse ESCs grew to about $70 \%$ of the cultivating bottle, they were dissociated and resuspended in media above without rmLIF. Then we seeded the individual drop of $30 \mathrm{ul}\left(0.5 \times 10^{5} / \mathrm{ml}\right)$ onto the surface of $100 \mathrm{~mm}$ bacterial grade plate and inverted the whole assembly. The handing-drop ESCs were incubated at $37{ }^{\circ} \mathrm{C}$ in $5 \% \mathrm{CO}_{2}$ for $2 \mathrm{~d}$. After incubation of $3 \mathrm{~d}$, the embryoid bodies (Ebs) were aspirated onto six-well tissue culture plates for adherent culture. Then several factors were sequentially added to the culture media for hepatocyte maturation as the following: $10^{-7} \mathrm{M}$ Dexamethasone (Dex) (Sigma) at d 1, 10ug/L fibroblast growth factor-4 (FGF-4) (Chemicon) at d 3 and $25 \mathrm{ug} / \mathrm{L}$ hepatocyte growth factor (HGF) (PeproTech) at d 5.

\section{Preparation of the conditional selective medium}

Cholestatic serum was prepared according to our previously reported method [15]. Sprague-Dawley rats weighing 200_250 g (Laboratory Animal Research Center of Sun Yat-sen University, Guangzhou, China) underwent ligation and transection of the common bile duct under general anesthesia with ether to induce cholestasis. Ten days after the operation, the rats were sacrificed and whole blood was collected. Serum was isolated from the whole blood and then subjected to liver function testing, inactivated, and sterilized for use in culture. Finally, cholestatic serum was added into the basic differentiating media mentioned above, to achieve $5 \%$ concentration. Differentiating media containing $5 \%$ cholestatic serum served as the conditional selective media. For the control group, the serum collected from normal rats was added into the basic differentiating media to achieve $5 \%$ concentration. In our study, primary differentiated hepatocytelike cells ( $9 \mathrm{~d}$ after initial induction) were transferred into the conditional selection media consisting of $5 \%$ cholestatic sera and cultured for 2 wks. Media was changed every $2 \mathrm{~d}$.

\section{Morphological changes}

Primary differentiated cells and selected cells were observed under inverted phase contrast microscope. Cells with regular multilateral shape were viewed as hepatocyte-like cells. What's more, $2 \mathrm{wk}$ after screening cultivation, ultrastructure of the cells was observed with electron microscopy.

\section{Immunohistochemistry assay}

$9 \mathrm{~d}$ after initial induction, differentiated ESCs were taken out and washed with PBS twice, then were fixed with $4 \%$ paraformaldehyde for $30 \mathrm{~min}$ at room temperature. Albumin and CK8/18 level were detected with polyclonal anti-mouse antibody for albumin (1:200) (Accurate Chemical) and polyclonal anti-mouse antibody for CK8/18 (1:200) (Lab Vision). The slides were incubated with respective primary antibodies at room temperature for $2 \mathrm{~h}$ in a humidified chamber. Blocking serum and biotinylated secondary antibody were matched with the primary antibody. Immunoperoxidase was stained and counterstained with DAB and Gill-I hematoxylin.

\section{$R T-q P C R$}

Total RNA was extracted using Trizol (invitrogen, USA) according to the product specification and the concentration of total RNA was measured with NanoDrop 2000c (Thermo, USA). 2 ug total RNA was obtained for cDNA synthesis with RevertAidTM First Strand cDNA Synthesis Kit (Fermentas, USA), Quantification of gene expression was performed using a real-time polymerase chain reaction (PCR) kit (Takara, Japan). The expression of the following genes was analyzed: ALB, TTR, AAT, G-6-P, CK18, TAT, FXR, Cyp7a1 and GAPDH (as internal reference). All primers used above were listed as follows: ALB sense: 5'- CAG GAT TGC AGA CAG ATA GTC-3' and antisense: 5'- GCT ACG GCA CAG TGC TTG-3'; G-6-P sense: 5'- CAG GAC TGG TTC ATC CTT-3' and antisense: 5'- GTT GCT GTA GTA GTC GGT-3'; TTR sense: 5'- CTC ACC ACA GAT GAG AAG-3' and antisense: 5'- GGC TGA GTC TCT CAA TTC-3' ; AAT sense: 5'- AAT GGA AGA AGC CAT TCG AT-3' and antisense: 5'- AAG ACT GTA GCT GCT GCA GC-3' ; CK18 sense: 5'-GGT ACT CTC CTC AAT CTG CTG-3' and antisense: 5'-CTC TGG ATT GAC TGT GGA AGT G-3' ; Transferrin sense: 5'-GCA GTG GCC AGT TTC TTC TC$3^{\prime}$ and antisense: 5'-TTA AAC AGC AGG TCC TTC CC-3' ; FXR sense: 5'-CCA ACC TGG GTT TCT ACC C-3' and antisense: 5'-CAC ACA GCT CAT CCC CTT T-3'; Cyp7a1 sense: 5'- ATG ATG ACT ATT TCT TTG ATT TGG GGA-

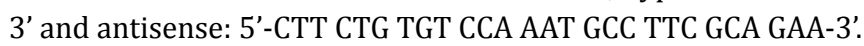


Immunofluorescence assay

$2 \mathrm{wk}$ after screening cultivation, the medium was removed. The selected hepatocyte-like cells were fixed in $4 \%$ paraformaldehyde in phosphate-buffered saline (PBS) at room temperature for $20 \mathrm{~min}$. Then they were blocked with PBS containing $0.1 \%$ Triton X-100, $10 \%$ normal horse serum and $1 \%$ bovine serum albumin for $1 \mathrm{~h}$. Cells next were incubated with the primary antibodies against mouse AFP, ALB (1:200) (Accurate Chemical), CK8/18 (1:200) (Lab Vision) and FXR (1:50)(R\&D Systems) at $4^{\circ} \mathrm{C}$ overnight. After 5 washes with PBS, biotinylated secondary antibody $(1: 100)$ was added and incubated at $37^{\circ} \mathrm{C}$ for $30 \mathrm{~min}$. At last, SABC-Cy3 $(1: 100)$ was added and incubated at $37^{\circ} \mathrm{C}$ for $30 \mathrm{~min}$. Cells were washed between each step with PBS containing $0.1 \%$ bovine serum albumin. As for the control group, the same amounts of cells were collected for immunofluorescence. Cytoplasm red was positive in the fluorescence microscopy.

\section{Glycogen dying assay}

2 wk after screening cultivation, the selected hepatocyte-like cells were fixed with $95 \%$ ethanol for 10 min and rinsed in $\mathrm{dH}_{2} \mathrm{O}$. Afterwards, cells were oxidized in $10 \mathrm{~g} / \mathrm{L}$ periodic acid for $15 \mathrm{~min}$, rinsed 3 times in $\mathrm{dH}_{2} \mathrm{O}$ and treated with Schiff's reagent for $30 \mathrm{~min}$, then rinsed in $\mathrm{dH}_{2} \mathrm{O}$ for $10 \mathrm{~min}$ again. At last, they were stained with Mayer's hematoxylin for 1 min and rinsed in $\mathrm{dH}_{2} \mathrm{O}$. Red granular deposits within cytoplasm with a light microscope were defined as the positive dying.

\section{Indocyanine Green (ICG) uptake assay}

Indocyanine green (ICG), a water-soluble dye that does not undergo metabolism or enterohepatic circulation, serves as a measure of hepatic function because of its exclusive elimination via biliary excretion [16]. $2 \mathrm{wk}$ after screening cultivation, selected hepatocyte-like cells were washed with PBS 3 times, $2 \mathrm{ml}$ ICG (Sigma) solution was added to the plates at a final concentration of $50 \mu \mathrm{M}$ and incubated at $37^{\circ} \mathrm{C}$ for $1 \mathrm{~h}$, then rinsed with PBS 3 times. Cytoplasm green was positive. The control group at the same period were not used the conditioning selection media. At last we randomly counted 100 cells and calculated the ICG positive cells, each group were repeatedly done in 6 cases. Positie results to ICG dyeing were displayed with mean + standard deviation.

\section{Assay of urea synthesis function and ammonia elimination}

Level of urea nitrogen can be used as an indicator of hepatocytes' function because of the urea synthesis capability of normal hepatocytes. Experiment was divided into five groups: mouse ESCs, cells induced for 9 days, cells induced for 23 days, cells after 2 wk screening cultivation and the group of normal hepatocytes. About $1 \times 10^{6}$ cells were taken respectively with 6 -well plates, and then discarded the medium; add $2 \mathrm{ml}$ culture medium, sucked out $10 \mu \mathrm{l}$ culture medium in the first three days for test. Colorimetric assay of cells' urea nitrogen were detected according to the urea nitrogen assay kit (Sigma, USA) instructions.

The detoxification capability of hepatocyte-like cells was evaluated by the ammonia elimination assay. Cells were divided into five groups as above and then incubated with serum-free DMEM containing $1 \mathrm{mmol} / \mathrm{L}$ ammonium chloride for 4 hours. The decrease in ammonia concentration in the medium during a $24 \mathrm{~h}$ period was measured using the automatic biochemistry analyzer (HITACH). We performed all tests including the controls in triplicate.

\section{CYP metabolism assay}

For the measurement of CYP enzyme activities, the screened cells and the control cells were cultured in the medium with $50 \mathrm{mM} 3$-methylcholanthrene for $48 \mathrm{~h}$. Cells were dissociated and incubated with substrate in $200 \mathrm{ml}$ incubation medium at different concentrations for $3 \mathrm{~h}$ at $37^{\circ} \mathrm{C}$. Then $800 \mathrm{ml}$ cold methanol was added and centrifuged to stop the reaction. The supernatants were collected for measurement of indicated productions by LC-MS/MS (Agilent 1200 HPLC and ABI 4000 massspectrometer). Normal rat hepatocytes were used as a positive control. Total cell protein amount was used to normalize the data. Substrates and metabolic products for standard were commercially purchased of phenacetin and diclofenac (Sigma).

Animal model establishment and intrahepatic transplantation of ESCs-derived hepatocyte-like cells labeled by CFDA-SE

Carboxy fluoresce in diacetate succinimidyl ester (CFDA-SE) (Molecular Probes) was used to label the transplanted cells. Firstly, 2 wk screening cultivation cells were digested and selected with $0.25 \%$ 
pancreatin and centrifuged at $1000 \mathrm{r} / \mathrm{min}$ for $5 \mathrm{~min}$. Then the supernatant was removed, and the cells were re-suspended in PBS containing $10 \mu \mathrm{mol} / \mathrm{L} \mathrm{CFDA-SE}$ and incubated at $37^{\circ} \mathrm{C}$ for $15 \mathrm{~min}$ to make sure the complete absorption of fluorescent tracer. Secondly, the obtained cells were centrifuged at $1000 \mathrm{r} / \mathrm{min}$ for 5 min and the supernatant was removed, then the cells were re-suspended in PBS and incubated at $37^{\circ} \mathrm{C}$ for another $30 \mathrm{~min}$. Acetyl group was removed from CFDA-SE under the action of esterase in plasma, and CFDA-SE was fluorescent.

$24 \mathrm{BALB} / \mathrm{c}$ mice, 6-8 wk old, weighing 20-35 g, irrespective of gender, clean grade (Guangzhou Experimental Animal Center) were selected. 12 random mice in the group A were intraperitoneally injected with $50 \mathrm{mg} / \mathrm{kg}$ retrorsine (Sigma) once every $2 \mathrm{wk}$ for totally twice. $4 \mathrm{wk}$ after the second injection, about $70 \%$ liver was resected. Then, about $1 \times 10^{5}$ hepatocyte-like cells selected by $5 \%$ cholestatic serum and labeled by CFDA-SE were transplanted into mouse liver through portal vein. On the other hand, $70 \%$ liver of another 12 mice in the group B was resected and the same ESCs-derived hepatocyte-like cells were transplanted into mouse liver. The liver samples were observed under fluorescent microscopy on wk 1 or 2 .

All mice were anesthetized with diethyl ether to expose the abdomens. Blood flow of portal vein was blocked for $15 \mathrm{~min}$ with forceps clip, and then fluorescence-labeled cells $\left(1 \times 10^{5}\right)$ were transplanted into mouse liver through portal vein.

\section{Albumin immunoassay after intrahepatic transplantation}

Differentiation of donor hepatocyte-like cells was determined in recipient liver with albumin fluorescence immunoassay (double fluorescence staining). Experimental procedures: $2 \mathrm{wk}$ after transplantation of ESCs-derived hepatocyte-like cells, 3 mice in each group were anesthetized and sacrificed. Liver tissue was rapidly obtained, frozen, and cut into serial sections ( $5 \mu \mathrm{m}$ in thickness). The frozen sections were fixed with 4\% formaldehyde, defrosted at room temperature for 15-30 min, and hydrated with PBS at room temperature in wet box for $15 \mathrm{~min}$. And then, the samples were added with PBS containing $0.2 \%$ Triton $\mathrm{X}-100$ (PBT) and maintained at room temperature for 20 min, added with PBT containing $0.2 \%$ BSA and $5 \%$ goat serum (or 1\% BSA) and maintained at room temperature for 30 min (to block non-specific binding), added with $1 \mu \mathrm{g}$ antibody 1 (rabbit-anti-mouse albumin monoclonal antibody dissolved in $20 \mu \mathrm{L} \mathrm{PBS}$ ) and $5 \mu \mathrm{L}$ antibody 2 (Zenon A reagent dissolved in antibody 1 solution) and incubated at room temperature for 5 min (albumin antibody was labeled by PE), and added with $5 \mu \mathrm{L}$ Zenon B reagent and incubated at room temperature for $5 \mathrm{~min}$ to block labeling reaction. PBT (3:1) was used to dissolve fluorescent-labeled albumin antibody (immune compound of antibodies 1 and 2). Tissue sections were dripped with fluorescent-labeled albumin antibody and incubated in dark slide at room temperature for 1.0-2.0 h. Subsequently, the samples were washed with PBT twice with each for 10-15 min and with PBS twice with each for $5 \mathrm{~min}$, re-fixed with $4 \%$ formaldehyde at room temperature for $15 \mathrm{~min}$, and washed with PBS. Finally, the samples were put under confocal fluorescent microscope to observe fluorescent signals.

\section{Liver function}

Two weeks after transplantation, blood of group A and B were taken by using routine biochemical check method (Hitachi) to measure serum albumin, total bilirubin, alanine aminotransferase (ALT) and aspartate aminotransferase(AST).

\section{Reliability evaluation}

Embryonic stem cells-derived hepatic stem cells were poured into liver of remedial liver regeneration mice, and undifferentiated embryonic stem cells were transplanted into subcutaneous tissue in axillary region as the controls to observe neoplastic formation in embryonic stem cells-derived hepatic stem cells over 3 months.

\section{Statistical analysis}

Statistical analysis was manually calculated by the first author. Measurement data were compared with $t$ test. 


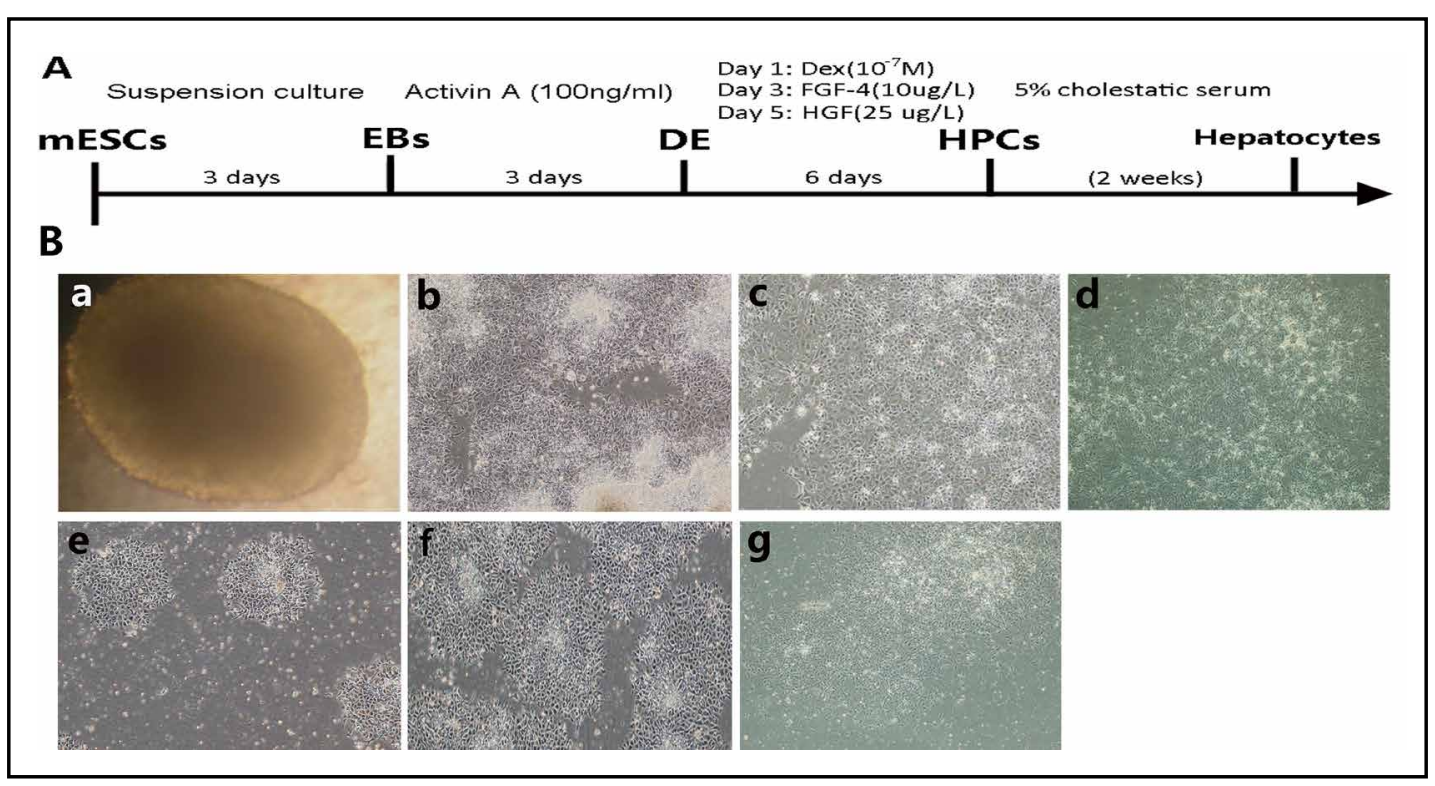

Fig. 1. Morphological change of initial differentiated and selected cells. (A)The procedure for differentiation of mouse ESCs into hepatocytes via EBs and DE is presented schematically. (B-a)The EBs presented as a ball clusters in suspension packed with semitransparent membrane within LIF-free suspension after 3 days culture. (B-b) $9 \mathrm{~d}$ after initial induction, a large number of hepatocyte-like cells were observed, which exhibited a characteristic of large nucleus and abundant cytoplasm outside of it, ranked as streak or Slablike structure,and had regular triangle or multilateral contours. (B-c) $2 \mathrm{~d}$ after screening cultivation in a conditioning selection media containing 5\% cholestatic sera, most cells were suffered from apoptosis or even death. (B-d) $4 \mathrm{~d}$ after screening cultivation in a conditioning selection media containing $5 \%$ cholestatic sera, most cells were suffered from serious apoptosis or even death. (B-e) $1 \mathrm{w}$ later, some hepatocyte-like colony-forming units (H-CFU) were observed and the cells showed good uniformity. (B-f) In 2 wk, there was a trend to mature from central to round area. Peripheral cells were more close to maturity liver cells in morphology. (B-g) After selected culture for $2 \mathrm{wk}$, few cells could survive in control group (undifferentiated E14 mouse ESCs). Magnifications: a e:100×; f, g: 40×.

\section{Results}

\section{Morphological observation}

The 3-day embryoid bodies (EBs) (Fig. 1 B-a) were transferred onto six-well tissue culture dishes for further cultivating. To realize the primary differentiation of ESCs into hepatic lineages, several growth factors (including FGF-4 and HGF) and Dex were sequentially added to culture media respectively. $9 \mathrm{~d}$ after initial induction, a large number of hepatocyte-like cells were observed, which exhibited a characteristic of large nucleus and abundant cytoplasm outside of it, ranked as streak or Slab-like structure, and had regular triangle or multilateral contours (Fig. 1 B-b). On the other hand, after screening cultivation in a conditioning selection media containing $5 \%$ cholestatic sera, most cells were suffered from apoptosis or even death in the early stage (Fig. 1 B-c and B-d), but $1 \mathrm{w}$ later, some hepatocyte-like colony-forming unit (H-CFU) were observed, in which most cells exhibiting hepatocellular morphological characteristics and the cells showed good uniformity (Fig. 1 $\mathrm{B}$-e). In $2 \mathrm{wk}$, there was a trend to mature from central to round area. Peripheral cells were more close to maturity liver cells in morphology (Fig. 1 B-f), while few cells could survive in control group (undifferentiated E14 mouse ESCs )(Fig. 1 B-g).

\section{Immunohistochemistry}

Firstly, we assessed these hepatocyte-like cells derived from ESCs by immuohistochemical assay. Both albumin and CK8/18 are the characteristic proteins expressed during hepatocyte 


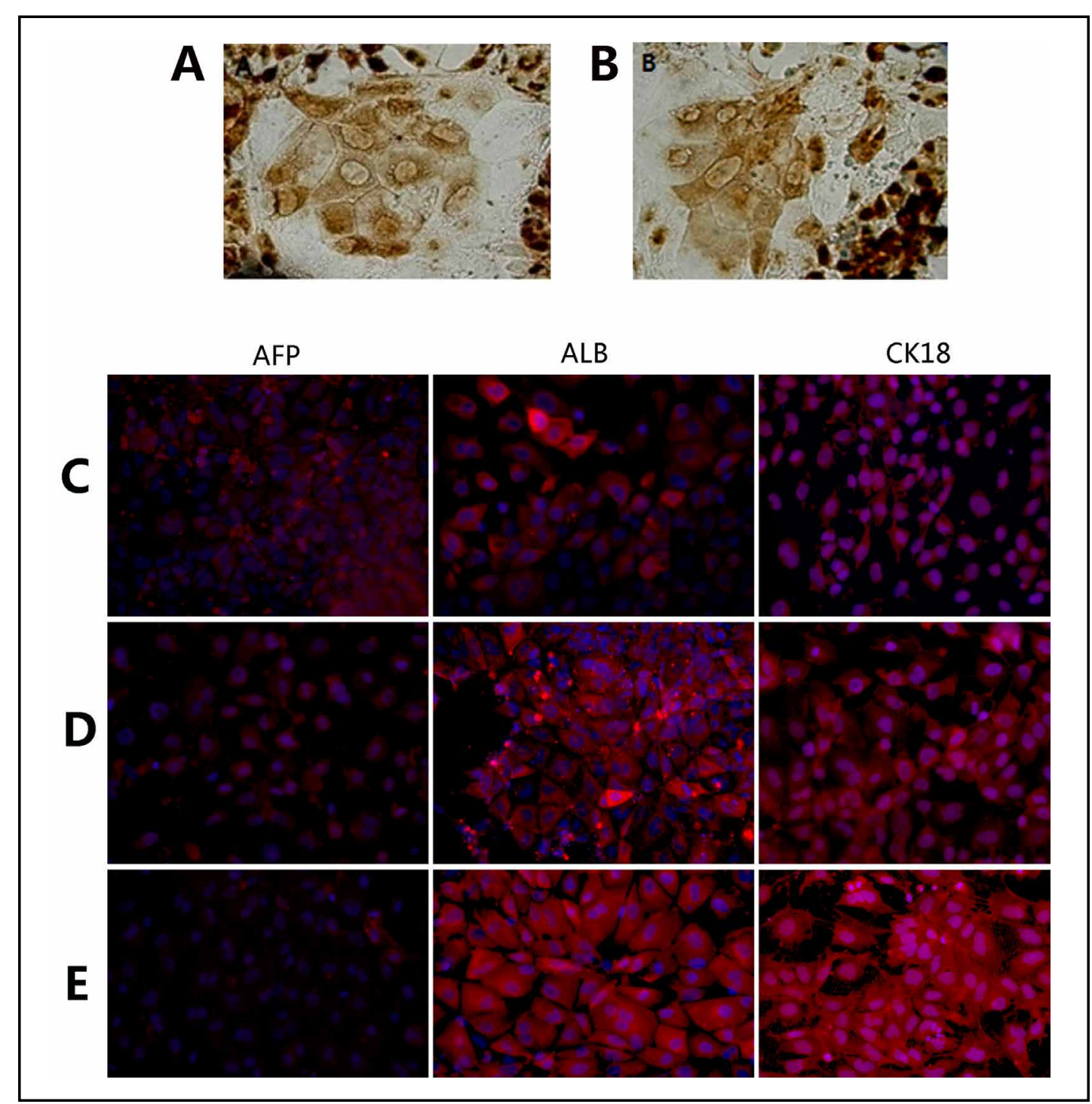

Fig. 2. Immunostaining of induced cells. Primary differentiated ESCs at day 9 were immunostained with anti-albumin antibody or anti-CK8/18 antibody followed by second antibody. Both albumin (A) and CK8/18 (B) positive staining (brown -yellow particals) were observed $(\times 400)$. (C): After 9 days of induction, ALB and CK18 protein in HPCs could be detected, but the express level was low, while AFP protein was highly expressed. (D): 23 days after induction, the levels of ALB and CK18 protein were little high comparing with 9d induction HPCs, while AFP was almost the same. (E): 2 wk after screening cultivation, cells were regular polygons with large nucleus and abundant cytoplasm in morphology. And the levels of ALB and CK18 protein were dramaticly increased than other two groups, while AFP protein fluorescence was almost invisible. Magnification: 200×. AFP: alpha fetoprotein; ALB: albumin; CK18: Cytokeratin 18.

development. $9 \mathrm{~d}$ after initial induction, the differentiated ESCs were immunostained with anti-albumin antibody or anti-CK8/18 antibody followed by second antibody. The results revealed brown-yellow particals were positive staining (Fig. 2 A and B). It explained that these cells had at least part hepatocyte function.

\section{Immunofluorescence}

9 days after induction, cells ALB and CK18 proteins were lowly expressed, while AFP protein was highly expressed (Fig. 2C). 23 days after induction(cells of the control groups), 

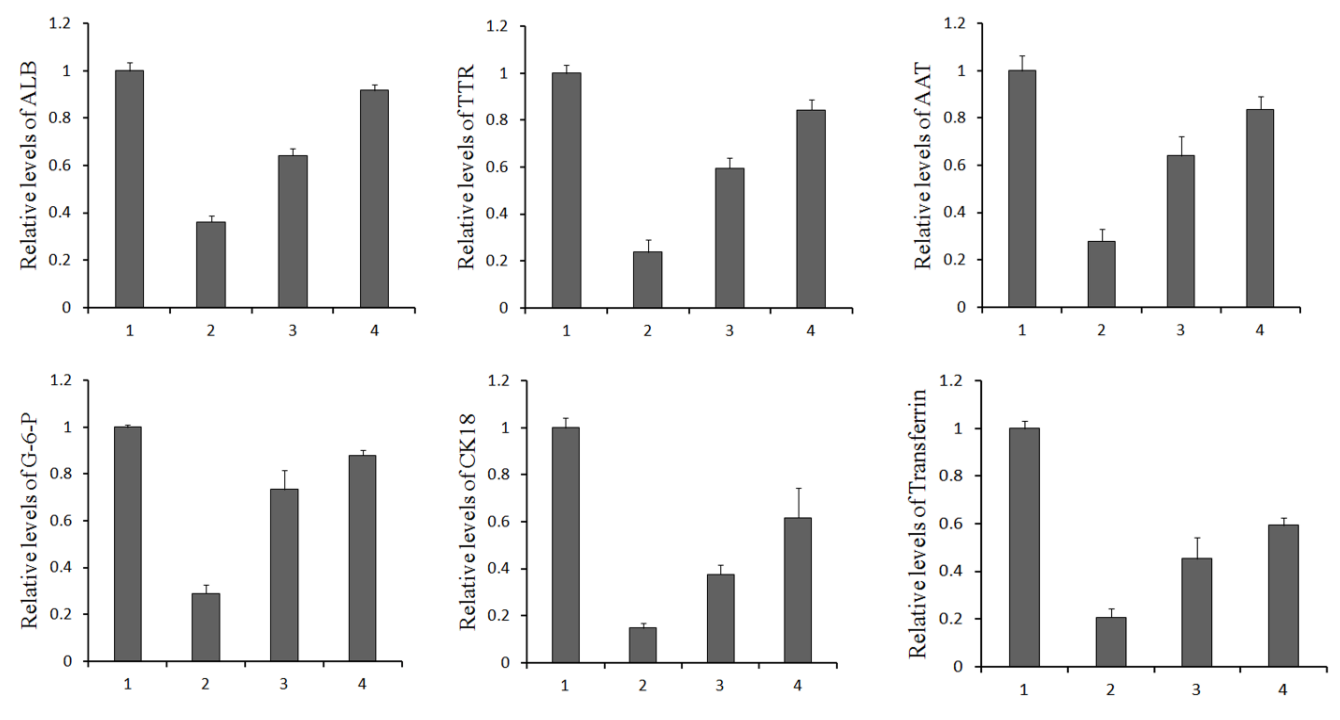

Fig. 3. Detection of the expression level of hepatocyte-specific genes by RT-qPCR. The mRNA levels of hepatocyte-specific genes on various induction stages. 1 represents the mouse mature hepatocytes, 2 for cells induced for 9 days, 3 on behalf of cells induced for 23 days, 4 represent cells undergoing 2 wk screening cultivation. The vertical axis stands for the ratio of the CT values of the 2-ddCt value of cells on various induction stages relative to mouse mature hepatocytes. It's shown that the level of hepatocyte-specific genes increased significantly after screening cultivation, especially on $6 \mathrm{~d}$, the level of ALB reach almost $90 \%$ of mature hepatocytes. T-tests were carried out respectively between group 3 and 4 , all the P values were less than 0.05. ALB: Albumin; TTR: transthyretin; AAT: Alanine Aminotransferase; G-6-P: glucose-6phosphatase. CK18: Cytokeratin 18.

the levels of ALB and CK18 protein were little high comparing with 9d induction HPCs, while AFP was almost the same (Fig. 2D). 2 wk after screening cultivation, cells were regular polygons with large nucleus and abundant cytoplasm in morphology, and the levels of ALB and CK18 protein were dramaticly increased than other two groups, while AFP protein fluorescence was almost invisible (Fig. 2E). This result revealed that after our screening cultivation, the selected cells could express hepatic protein highly.

\section{Detection of the expression of hepatic special genes by RT-qPCR}

mRNA levels of hepatocytes special genes expression, containing ALB, TTR, AAT, G-6-P, CK18, TAT were detected by real-time PCR. 9 days after induction, the HPCs can express the hepatocytes special genes listed above, but level was very low. Comparing with control group cells, the mRNA levels of hepatocytes special gens of experimental group cells which undergoing 2 wk screening cultivation were significantly increased, what's more they were close to the level of normal liver cells (Fig. 3), especially the level of ALB reached $90 \%$ level of mouse mature hepatocytes. From this result, we can conclude that our screening cultivation can promote the express of hepatocytes special gens of $9 \mathrm{~d}$ induction HPCs.

ICG uptake, glycogen dying, urea synthesis function tests, ammonia elimination and CYP metabolism assay

23 days after induction, we examined ICG uptake, which is a liver-specific function to identify differentiated hepatocytes in vitro. These cells took up ICG from the medium and showed little green, while after 2 wk screening cultivation cells of the test group showed more dark green, which indicated that the selected cells had better function of ICG uptaking (Fig. 4A). Meanwhile, glycogen dying was detected after 23 days after induction. Little 


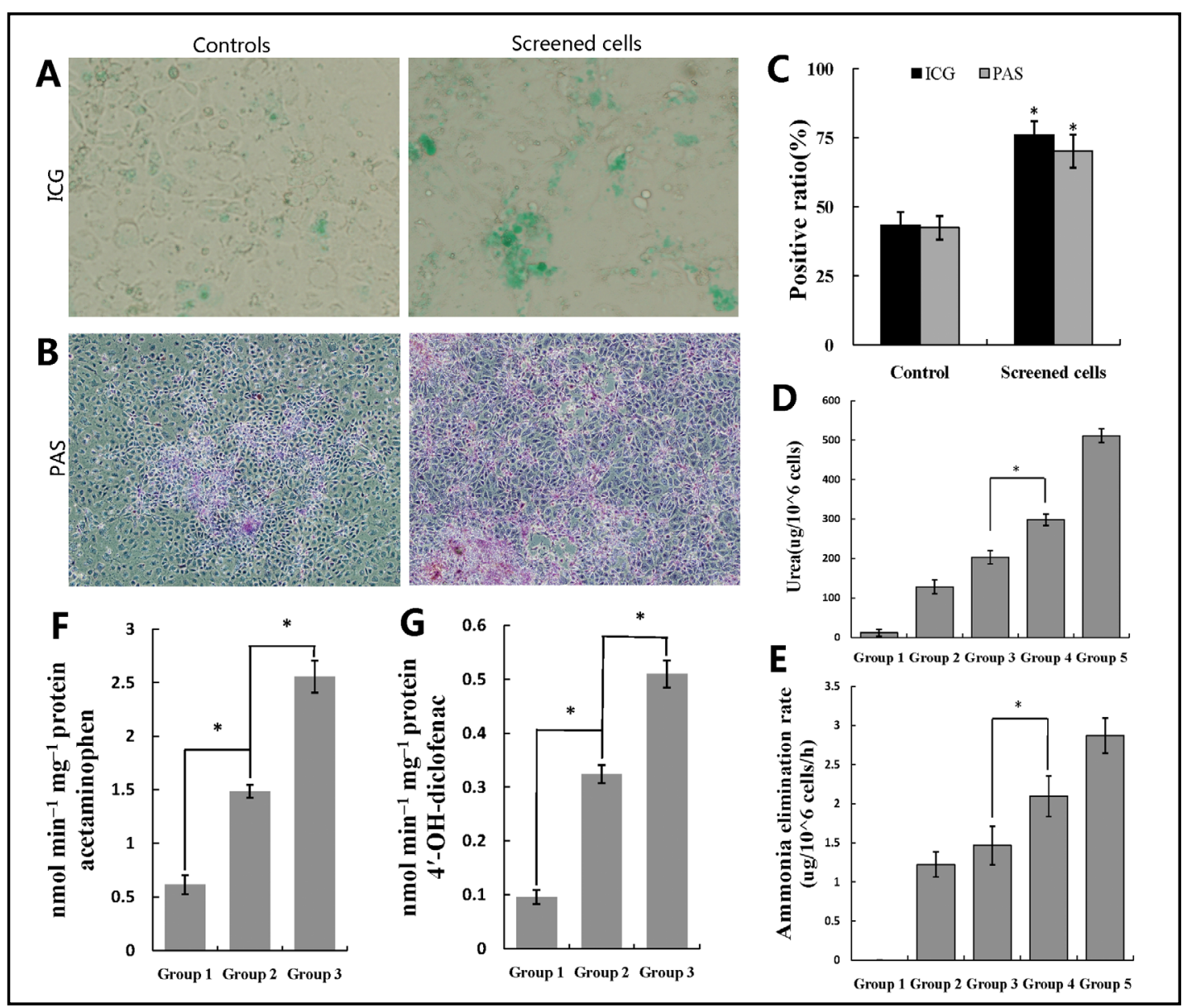

Fig. 4. Detection of the hepatic function of the induced cells. ICG uptake test and glycogen dying assay. (A): 23 days after induction, some cells could take up ICG and show little green. $2 \mathrm{wk}$ after screening cultivation, most cells took up ICG from the medium and showed dark green. The backgroud colors of the two pictures were deleted. (B): Red granular deposits within cytoplasm indicated the hepatocyte-like cells of test group had the function of glycogen synthesis, the control group expressed little glycogen staining. The experimental group expressed much more glycogen staining. (C): The average ICG uptake and glycogen dying ratio of experimental groups were $76.13 \%$ and $70.22 \%$ respectively; while the control groups' were $43.43 \%$ and $42.47 \%$ respectively $(P<0.05$ both in ICG and PAS). (D): Urea synthesis function was detected in five groups: Group 1 stands for mouse ESCs; Group 2 represent mouse ESCs induced for 9 days. Group 3 stands for the ESCs induced for 23 days and Group 4 stand for cells after 2 wk screening cultivation, Group 5 for mature mouse hepatocytes. ${ }^{*} P<0.05$. (E) For ammonia removal rate, cells were divided into 5 groups as urea synthesis. The ammonia removal activity of rat ESCs couldn't be detected. As the induction going on, it was gradually increased. After $2 \mathrm{wk}$ screening cultivation, the ammonia removal activity was $2.09 \pm 0.25 \mu \mathrm{g} / 10^{6}$ cells per hour, higher than the control group and reach to $70 \%$ of mature mouse hepatocytes. (F, G): CYP metabolic activities of the induced cells and normal rat hepatocyte. Group 1 stand for the control group cells, Group 2 for the cells after 2 wk screening cultivation, Group 3 for the normal rat hepatocyte. The metabolic products of phenacetin (converted to acetaminophen by Cyp1a2; F) and diclofenac (converted to 4'-OHdiclofenac by Cyp2c enzymes; G) were determined by liquid chromatography-tandem mass spectrometry according to standard curve. ${ }^{*} P<0.05$. The above results indicated that miR-122 could promote the functional maturation of HPCs. Magnification: $100 \times$.

expression of glycogen staining in control group was detected, whereas much more red granular deposits within cytoplasm were observed in the experimental groups, indicating that the cells transfected with miR-122 had better function of glycogen synthesis (Fig. 4B). In 


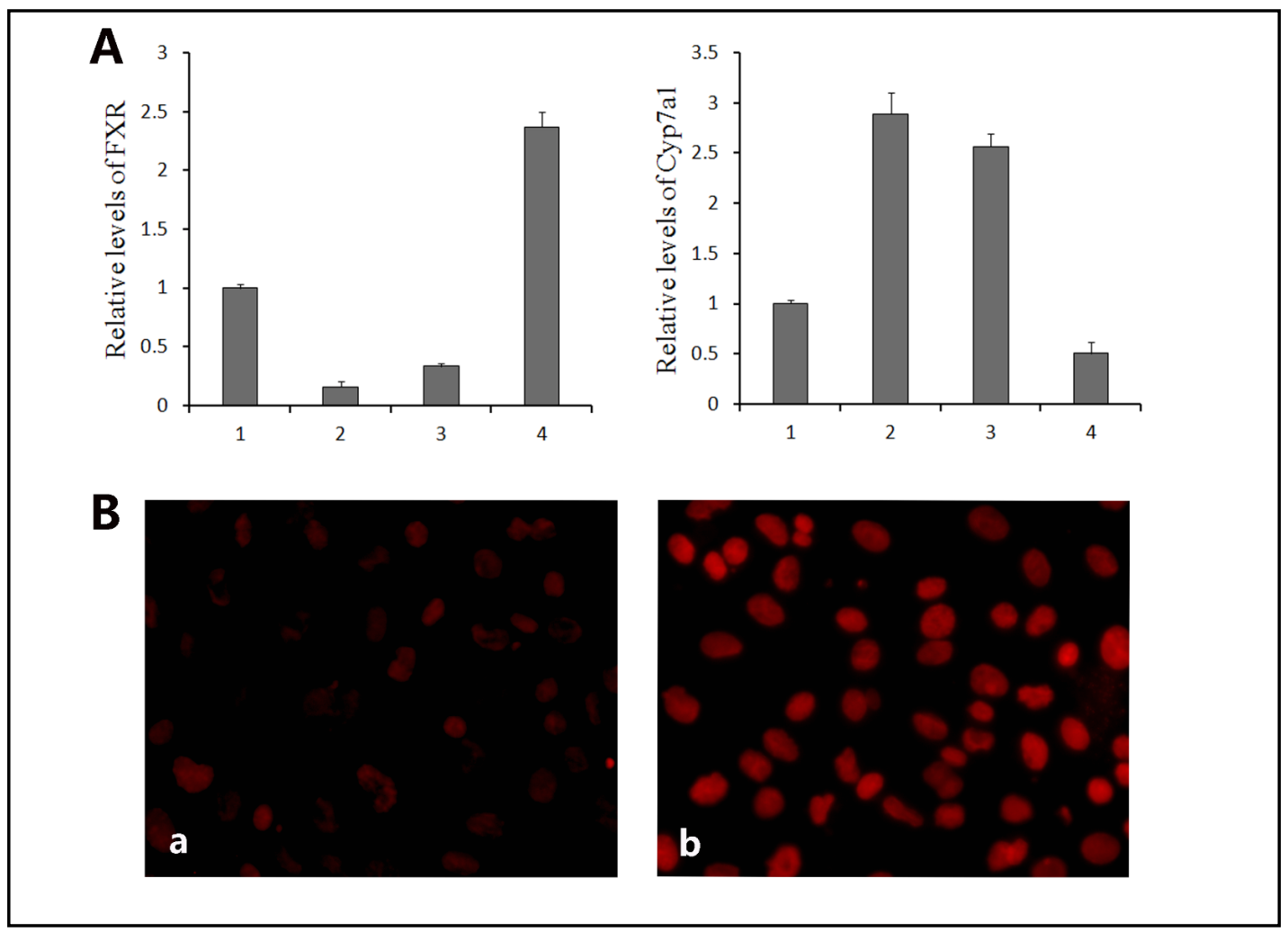

Fig. 5. Hepatic FXR and Cyp7a1 expression levels induced and screening cultivated ESCs. Both FXR and Cyp7a1 mRNA was measured by quantitative PCR in four groups (A): 1 represents the mouse mature hepatocytes, 2 for cells induced for 9 days, 3 on behalf of cells induced for 23 days, 4 represents cells undergoing $2 \mathrm{wk}$ screening cultivation. Data are expressed as means \pm SD. After screening cultivation, the FXR expression of hepatocyte-like cells was increased while its negative target gene Cyp7a1 was decreased. T-test was indicated between group 3 and $4(P<0.05)$. (B): The FXR protein level was detected by immunofluorescence, a stands for cells induced for 23 days, b represents cells undergoing 2 wk screening cultivation.

addition, both the ICG-positive and PAS-positive cells number of test group were higher than the control by statistic analysis (Fig. 4C). With the induction going on, the urea synthesis level was gradually increased. After 2 wk screening cultivation, urea synthesis function of cells was significantly increased, and urea synthesis level reached almost $70 \%$ of mouse normal liver cells (Fig. 4D). Similarly, the ammonia elimination rates tend to rise with culture time. After 2 wk screening cultivation, the ammonia removal rate was $2.09 \pm 0.26 \mu \mathrm{g} / 10^{6} \mathrm{cells}$ per hour, while the control group was $2.09 \pm 0.25 \mu \mathrm{g} / 10^{6}$ cells per hour (Fig. 4E). Ammonia removal activity in differentiating ES cells was detected by day 21 and increased with culture time. Finally, the CYP metabolism activities of the control cells, screened cells and normal rat hepatocytes were detected by determining the metabolic products of phenacetin and diclofenac (Fig. 4F,G). The above results indicated that our screening cultivation could promote the functional maturation of HPCs.

\section{Hepatic FXR gene expression is increased after screening cultivation}

Recent investigations have revealed that bile acids (BA) may be essential in the initiation of the hepatoproliferative response via farnesoid X receptor (FXR) stimulation [17]. To determine whether FXR gene expression is regulated after induction and screening cultivation, hepatic FXR mRNA levels were measured by RT-qPCR. A significant increment of hepatic FXR mRNA expression was observed in screening cultivated cells compared with control group. To evaluate whether increased expression of hepatic FXR mRNA after 

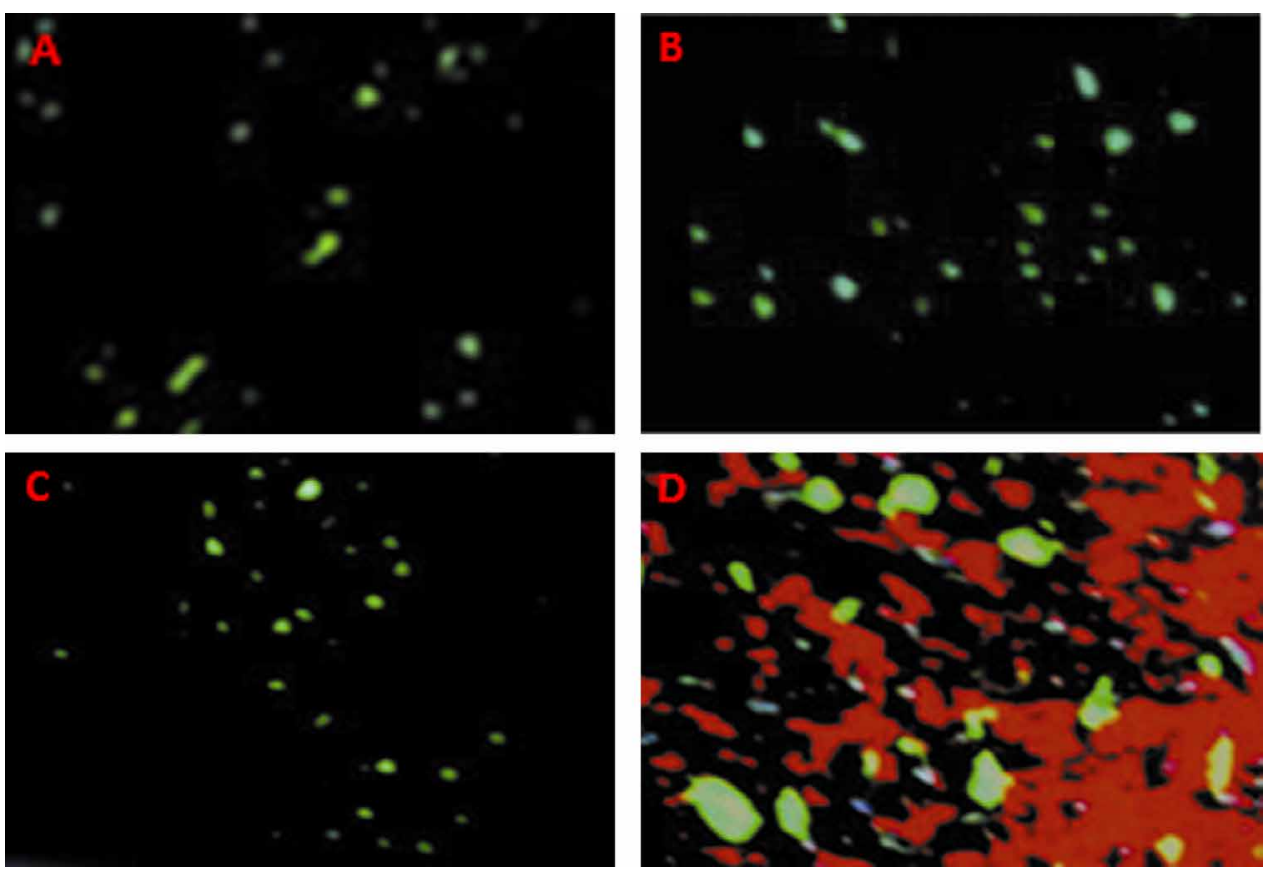

Fig. 6. Distribution of CFDA-SE-labeled cells in recipient mouse liver after transplantation. (A) 1 wk posttransplantation, some scattered region was green in hepatic parenchyma under fluorescent microscopy in both groups $(\times 200)$. (B) In the liver regeneration model + hepatocyte-like cells transplantation group, the area of green region was enlarged apparently in $2 \mathrm{wk}$, and hepatic cord-like structure could be observed $\operatorname{dimly}(\times 200)$. (C) In the partial liver resection + hepatocyte-like cells transplantation group, the area of green region was not apparently enlarged as compared in the liver regeneration model + stem cell transplantation group, but hepatic cord-like structure could also be observed $(\times 200)$. (D) 2 wk post-transplantation, immunofluorescent staining of albumin (double staining) of liver tissue demonstrated some positive cells, which showed yellow $(\times 200)$.

induction and screening cultivation is associated with altered FXR target gene expression, Cyp7a1 expression was analyzed. Liver Cyp7a1, a negative target of FXR [18], expression tended to decreased screening cultivated cells (Fig. 5A). Demonstration of FXR protein by immunofluorescence microscopy also confirmed that FXR expression was increased (Fig. 5B) after screening cultivation. These results infer the FXR pathway may be an important factor for liver cells differentiation and development.

\section{Intrahepatic distribution and function of CFDA-SE-labeled cells after transplantation}

$1 \mathrm{wk}$ after transplantation of CFDA-SE-labeled ESCs-derived hepatocyte-like cells in recipient mice, scattered green fluorescence was distributed in hepatic parenchyma in both groups, and density was generally coincident (Fig. 6A). 2 wk later, scattered green fluorescence was enlarged in hepatic parenchyma in the liver regeneration model + hepatocyte-like cells transplantation group, and the scattered green fluorescence arrayed like hepatic cord structure (Fig. 6B). The area of scattered green fluorescence in the partial liver resection + hepatocyte-like cells transplantation group was not significantly enlarged as compared in the liver regeneration model + hepatocyte-like cells transplantation group, but a hepatic cord-like structure was still observed (Fig. 6C). Normal liver tissue was red under fluorescent microscopy, while CFDA-SE-labeled cells were green under fluorescent microscopy. Besides, 2 wk after transplantation of CFDA-SE-labeled cells, the albumin fluorescent immunohistochemistry (double staining) demonstrated that labeled cells could 
A

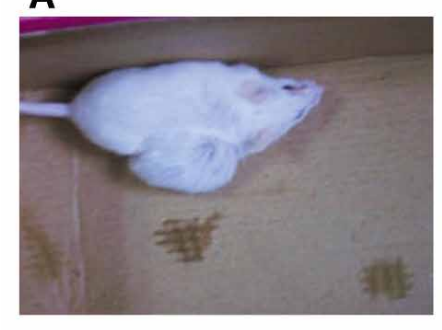

B

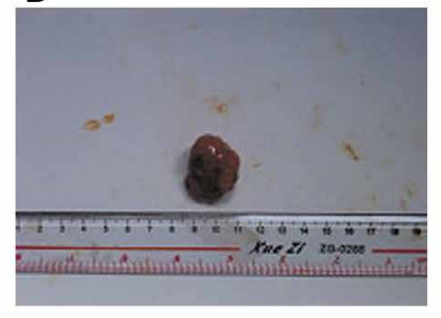

E

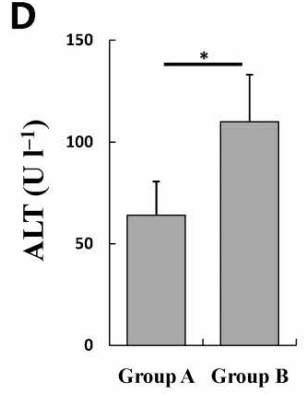

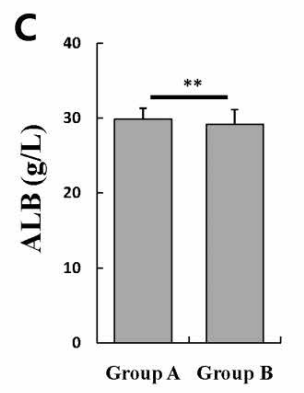

$\mathbf{F}$

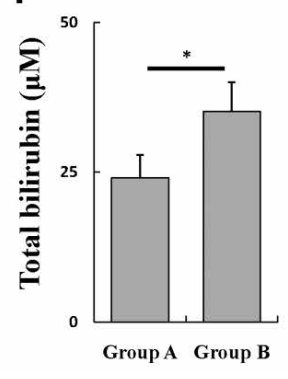

Fig. 7. Teratoma formation caused by subcutaneously transplanting undifferentiated embryonic stem cells in axillary region. (A): A large teratoma was observed in axillary region in 6 weeks post-transplantation, which was implanted with undifferentiated embryonic stem cells. (B): The volume of teratoma is about $2.5 \mathrm{~cm} \times 2 \mathrm{~cm} \times 2 \mathrm{~cm}$. However, pouring hepatocyte-like cells selected by a pathological microenvironmental culture system consisting of cholestatic sera into therapeutic liver repopulation model mice could not cause the formation of teratoma during 3 months. (C F): Serumlevels of albumin (C), ALT (D), AST (E) and total bilirubin $(\mathrm{F})$, in group A and group $\mathrm{B}(\mathrm{n}=5)$. ${ }^{*} P<0.05,{ }^{* *} P>0.05, t$-test. Data are presented as mean \pm s.d.

express positive signals of albumin in liver tissue of recipient mice in the two groups (Fig. 6D), which showed yellow. However, amount of positive signals in the liver regeneration model + hepatocyte-like cells transplantation group was apparently higher than in the partial liver resection + hepatocyte-like cells transplantation group.

\section{Liver function}

Two weeks after transplantation of hepatic stem cells, serum albumin levels were $(29.9 \pm 1.45) \mathrm{g} / \mathrm{L}$ in the group $\mathrm{A}$ and $(29.2 \pm 1.93) \mathrm{g} / \mathrm{L}$ in the group $\mathrm{B}$, and there was no significant difference between the two groups $(P>0.05)$ (Fig. 7 C). However, all of the total bilirubin, ALT and AST levels of group A were lower than group B $(P<0.05)$ (Fig. 7 D,E,F). This results suggested that the liver function of group $A$ was better than group $B$.

\section{Reliability evaluation}

Transplantation of undifferentiated embryonic stem cells in the axillary region could cause formation of teratoma after 6 weeks (Fig. 7 A and B); however, pouring hepatocyte-like cells selected by a pathological microenvironmental culture system consisting of cholestatic sera into therapeutic liver repopulation model mice could not cause the formation of teratoma during 3 months.

\section{Discussion}

Although ESCs can differentiate into hepatocyte-like cells either spontaneously or after induction in vitro, the resulting cells still contain multiple heterogeneous lineages which are 
not suitable for therapeutic transplantation. To avoid the risk of teratoma formation after cell transplantation, recent reasearch highlights the importance of acquiring functional ESCs-derived hepatocyte with a uniform phenotype very much.

In this study, we first prepared embryoid bodies for the purpose to increase the interaction of ESCs and create 3 levels of mesoderm in vitro. Then we induced the ESCs to differentiate into hepatocytes with Dex, FGF-4 and HGF sequentially. The exogenous factors we selected for promoting hepatic differentiation are in accordence with the liver developmental biology research. Zaret KS et al. found that FGFs was the crucial factor to activate the liver specific gene expression and could elicit embryonic induction of the liver from the mammalian gut endoderm [19]. Hepatocyte growth factor (HGF), as a potent mitogen for hepatocytes, shows mitogenic, motogenic and morphogenic activities for a wide variety of cells that express the HGF receptor c-Met, a transmembrane protein possessing an intracellular tyrosine kinase domain $[20,21]$. Dexamethasone (Dex) has the function to up-regulate GATA-4, HNF$3 \beta$ and Hex during progress from endoderm to ventral foregut, besides this, it also could promote HNF-4 $\alpha$ expression [22]. Our results showed that the EBCs could differentiate into hepatocyte-like cells $9 \mathrm{~d}$ after initial induction with Dex, FGF-4 and HGF, which exhibited a characteristic of large nucleus and abundant cytoplasm outside of it, ranked as streak or Slablike structure, and had regular triangle or multilateral contours (Fig. 1). The immunostaining (Fig. 2), RT-PCR results (Fig. 3) and the hepatic-function test (Fig. 4) revealed that the ESCsderived hepatocyte-like cells not only had the morphological character, but also expressed at least partial hepatocyte function. In short, our initial induction was effective.

At present, technologies used to screen hepatocyte-like cells from differentiated ESCs mainly include: Flow Cytometry cell sorting and magnetic activated cell sorting. However, all the methods mentioned above are either complex or have poor isolation efficiency, mainly because no very specific surface marker of hepatocyte-like cells had been found so far. Our team had reported that a conditioning selection media containing $5 \%$ cholestatic serum could select and purify the hepatic stem cells from bone marrow cells in vitro [15]. Here, we adopted the similar culture system containing $5 \%$ cholestatic serum to detect if it could select hepatocyte-like cells from differentiated ESCs initially induced with Dex, FGF-4 and HGF. Our results showed that most cells had been suffered from apoptosis or even death in the early stage (Fig. 1 B-c and B-d), but $1 \mathrm{w}$ later, some hepatocyte-like colony-forming unit (H-CFU) had been observed, in which most cells exhibited hepatocellular morphological characteristics and showed good uniformity (Fig. 1 B-e), while few cells could survive in control group (undifferentiated E14 mouse ESCs ) (Fig. 1 B-g). Indocyanine Green (ICG) uptake and PAS were positive for most of cells, and the positive ratio of experimental group was significantly higher than that of control group. The results of urea synthesis function tests, ammonia elimination and CYP metabolism assay also demonstrate that our selected cells have better hepatocyte function (Fig. 4). It proved our selection media containing $5 \%$ cholestatic serum could effectively select and purify the hepatocyte-like cells from differentiated ESCs in vitro.

Previous experiments had indicated the cholestatic serum contained humoral factors that had a role in recruiting putative liver stem cells and induced them to proliferate and differentiate [23]. Our previous research has demonstrated that the conditional selective medium containing cholestatic serum is optimal to selectively enrich hepatocyte-like cells from mixed differentiated ESCs [24]. Here, we consider the mechanism may be as following: In the context of cholestasis, organism will produce some humoral signal factors reactively through self-regulation to promote liver regeneration. So, there are some beneficial ingredients for liver regeneration in the cholestatic serum, such as phospholipids, cholesterol and some growth factors. On the other hand, cholestatic serum contains some toxic metabolites, such as bilirubin, cholalic acid, endotoxin, ammonia and aromatic amino acids; and these pathological toxic products are only metabolized through liver cells. For example, the hepatocyte-like cells expressing glucuronyl transferase can combine with bile acid and clear away bilirubin and synthetize urea to remove ammonia. When cultured in the cholestatic serum with appropriate concentration, only the functional hepatocyte-like 
cells could dispose these toxic metabolites, survive and then selectively proliferate and differentiate in response to the signals characteristic of cholestatic serum, while the nonhepatic cells could not adapt to such a pathological environment and result in apoptosis and death. Therefore, we think the pathological serum containing $5 \%$ cholestatic serum has two function: First, it activates hepatocyte-like cells selective proliferation and differentiation signals. Second, it washes out the non-hepatocyte-like cells.

The farnesoid X receptor (FXR, also named RIP14and HRP1), which belongs to a subcluster of metabolic receptors, could bind to DNA either as a monomer or as a heterodimer with a common partner for NRs or retinoid X receptor (RXR) to regulate the expression of various genes involved in bile acid (BA), lipid and glucose metabolisms [25]. Many ligands can activate the FXR and regulate the expression of various genes [26, 27]. Wang et al. found that activation of FXR by BAs increased the expression of FoxM1b, which is shown to regulate cell cycle progression during liver regeneration, implying that the FXR is a mediator of the effect of BA signaling on liver regeneration [28]. The FXR is a nuclear receptor highly expressed in the liver, intestine, adrenal, kidney [29]. FXR binds to various FXR response elements (FXREs) in different manners [30,31]. Upon activation by bile acids (BA), its endogenous ligands [32], FXR down-regulates BA synthesis via suppressing the rate-limiting enzyme cholesterol 7a hydroxylase (Cyp7a1) in order to prevent BA overload and toxicity [33]. Previous data have also confirmed the essential roles of BA and FXR in lipid metabolism [34-36]. In addtion the modulation of BA flux seems essential for liver regeneration to proceed normally [37]. Overall, bile acid-activated FXR controls bile acid homeostasis. Our result also comfirmed the expression of FXR was significantly increased after screening cultivation with BA, and its negative target gene Cyp7a1 was decreased (Fig. 5). Based on the composition of cholestatic serum and our results, we infer the FXR pathway may also be one important factor for liver cells differentiation and development.

Therapeutic liver repopulation (TLR) by transplanted hepatocytes is a promising approach for many inborn errors of metabolism and it has a number of potential advantages over whole organ transplant $[38,39]$. Under normal circumstances, hepatocytes of recipient itself proliferates precedently, so transplanted hepatocytes are not able to proliferate to reach the required amount. Otherwise, if retrorsine (RS) is used to pre-inhibit proliferation of recipient hepatocytes, followed by applying exogenous factors which can promote growth and differentiation of hepatocytes or stimulating excretion of exogenous factors through inducing liver injury, transplanted hepatocytes will be promoted to proliferate and nearly completely replace original hepatocytes [40-43]. In this study, we also adopted the similar way to establish therapeutic liver repopulation models for estimating the transplanted hepatocyte-like cells selected with a conditioning selection media consisting of $5 \%$ cholestatic sera. To be optimistic, our results demonstrated that these cells could effectively integrate into hepatic plate of host, further grow, differentiate, persist partial hepatic function and would not cause the formation of teratoma during 3 months (Fig. 6, 7). Our study explained the reliability and security of these selected ESCs-derived hepatocyte-like cells.

In summary, we established a simple and efficient method to select hepatocyte-like cells from differentiated ESCs.Our conditioning selection media consisting of 5\% cholestatic serum could not only effectively select and purify hepatocyte-like cells from differentiated ESCs initially induced by Dex, FGF-4 and HGF, but also promote the growth and differentiation of hepatocyte-like cells. After intrahepatic transplantation in therapeutic liver repopulation models, the selected cells could further grow, differentiate and persist partial hepatic function without forming teratoma. But, there is still a long way to go if we want to rebuilt a liver organ with ESCs-derived hepatocyte-like cells.

\section{Funding}

The National Natural Science Foundation of China (No. 30872700, 30471799) and the Natural Science Foundation of Guangdong Province(No. 07001617). 


\section{References}

1 Akhter J, Johnson LA, Gunasegaram A, Riordan SM, Morris DL: Hepatocyte transplantation: a review of laboratory techniques and clinical experiences. Surgeon 2007;5:155-164.

-2 Sundback CA, Vacanti JP: Alternatives to liver transplantation: from hepatocyte transplantation to tissueengineered organs. Gastroenterology 2000;118:438-442.

- 3 Nussler A, Konig S, Ott M, Sokal E, Christ B, Thasler W, Brulport M, Gabelein G, Schormann W, Schulze M, Ellis E, Kraemer M, Nocken F, Fleig W, Manns M, Strom SC, Hengstler JG: Present status and perspectives of cellbased therapies for liver diseases. Journal of Hepatology 2006;45:144-159 .

4 Strom S, Fisher R: Hepatocyte transplantation: new possibilities for therapy. Gastroenterology 2003;124:568571.

5 Thomson JA, Itskovitz-Eldor J, Shapiro SS, Waknitz MA, Swiergiel JJ, Marshall VS, Jones JM: Embryonic stem cell lines derived from human blastocysts. Science 1998;282:1145-1147.

-6 Kubo A, Kim YH, Irion S, Kasuda S, Takeuchi M, Ohashi K, Iwano M, Dohi Y, Saito Y, Snodgrass R, Keller G: The homeobox gene Hex regulates hepatocyte differentiation from embryonic stem cell-derived endoderm. Hepatology 2010;51:633-641.

7 Cai J, Zhao Y, Liu Y, Ye F, Song Z, Qin H, Meng S, Chen Y, Zhou R, Song X, Guo Y, Ding M, Deng H: Directed differentiation of human embryonic stem cells into functional hepatic cells. Hepatology 2007;45:1229-1239.

$>8$ Moore RN, Dasgupta A, Rajaei N, Yarmush ML, Toner M, Larue L, Moghe PV: Enhanced differentiation of embryonic stem cells using co-cultivation with hepatocytes. Biotechnology and Bioengineering 2008;101:1332-1343.

9 Ishii T, Fukumitsu K, Yasuchika K, Adachi K, Kawase E, Suemori H, Nakatsuji N, Ikai I, Uemoto S: Effects of extracellular matrixes and growth factors on the hepatic differentiation of human embryonic stem cells. American Journal of Physiology - Gastrointestinal and Liver Physiology 2008;295:G313-G321.

10 Baharvand H, Hashemi SM, Shahsavani M: Differentiation of human embryonic stem cells into functional hepatocyte-like cells in a serum-free adherent culture condition. Differentiation 2008;76:465-477.

11 Choo AB, Tan HL, Ang SN, Fong WJ, Chin A, Lo J, Zheng L, Hentze H, Philp RJ, Oh SKW, Yap M: Selection against undifferentiated human embryonic stem cells by a cytotoxic antibody recognizing podocalyxin-like protein-1. Stem Cells 2008;26:1454-1463.

12 Yamada T, Yoshikawa M, Kanda S, Kato Y, Nakajima Y, Ishizaka S, Tsunoda Y: In vitro differentiation of embryonic stem cells into hepatocyte-like cells identified by cellular uptake of indocyanine green. Stem Cells 2002;20:146-154.

13 Yin Y, Yew KL, Salto-Tellez M, Ng SC, Lin CS, Lim SK: AFP(+), ESC-derived cells engraft and differentiate into hepatocytes in vivo. Stem Cells 2002;20:338-346.

14 Drobinskaya I, Linn T, Saric T, Bretzel RG, Bohlen H, Hescheler J, Kolossov E: Scalable selection of hepatocyteand hepatocyte precursor-like cells from culture of differentiating transgenically modified murine embryonic stem cells. Stem Cells 2008;26:2245-2256.

15 Yun-Feng Cai, Zuo-Jun Zhen, Jun Min, Fang TL, Chu ZH, Chen JS: Selection, proliferation and differentiation of bone marrow-derived liver stem cells with a culture system containing cholestatic serum in vitro. World J Gastroenterol 2004;10:3308-3312.

16 Cherrick GR, Stein SW, Leevy CM, Davidson CS: Indocyanine green: observations on its physical properties, plasma decay, and hepatic extraction. J Clin Invest 1960;39:592-600.

-17 Huang W, Ma K, Zhang J, Qatanani M, Cuvillier J, Liu J, Dong B, Huang X, Moore DD: Nuclear receptordependent bile acid signaling is required for normal liver regeneration. Science 2006;312:233-236.

18 Zhang L, Huang X, Meng Z, Dong B, Shiah S, Moore DD, Huang W: Significance and mechanisms of CYP7a1 gene regulation during the acute phase of liver regeneration. Mol Endocrinol 2009;23:137-145.

19 Zaret KS: Hepatocyte differentiation: From the endoderm and beyond. Current Opinion in Genetics and Development 2001;11:568-574.

20 Rubin JS, Bottaro DP, Aaronson SA: Hepatocyte growth factor/scatter factor and its receptor, the c-met protooncogene product. Biochimica et Biophysica Acta - Reviews on Cancer 1993;1155: 357-371.

21 Zarnegar R, Michalopoulos GK: The many faces of hepatocyte growth factor: From hepatopoiesis to hematopoiesis. J Cell Biol 1995;129:1177-1180.

22 Li J, Ning G, Duncan SA: Mammalian hepatocyte differentiation requires the transcription factor HNF-4alpha. Genes Dev 2000;14:464-474. 
23 Avital I, Inderbitzin D, Aoki T, Tyan DB, Cohen AH, Ferraresso C, Rozga J, Arnaout WS, Demetriou AA: Isolation, characterization, and transplantation of bone marrow-derived hepatocyte stem cells. Biochem Biophys Res Commun 2001;288:156-164.

24 Min J, Shang CZ, Chen YJ, Zhang L, Liu L, Deng XG, Yang M, Chen DP, Cao J, Song EW, Chen JS: Selective enrichment of hepatocytes from mouse embryonic stem cells with a culture system containing cholestatic serum. Acta Pharmacol Sin 2007;28:1931-1937.

25 Wang YD, Chen WD, Huang W: FXR, a target for different diseases. Histol Histopathol 2008;23:621-627.

26 Zhao A, Yu J, Lew JL, Huang L, Wright SD, Cui J: Polyunsaturated fatty acids are FXR ligands and differentially regulate expression of FXR targets. DNA and Cell Biology 2004;23:519-526.

27 Nishimaki-Mogami T, Une M, Fujino T, Sato Y, Tamehiro N, Kawahara Y, Shudo K, Inoue K: Identification of intermediates in the bile acid synthetic pathway as ligands for the farnesoid X receptor. J Lipid Res 2004;45:1538-1545.

-28 Wang X, Krupczak-Hollis K, Tan Y, Dennewitz MB, Adami GR, Costa RH: Increased hepatic Forkhead Box M1B (FoxM1B) levels in old-aged mice stimulated liver regeneration through diminished p27Kip1 protein levels and increased Cdc25B expression. J Biol Chem 2002;277:44310-44316.

29 Chiang JY: Bile acid regulation of gene expression: roles of nuclear hormone receptors. Endocr Rev 2002;23:443-463

-30 Laffitte BA, Kast HR, Nguyen CM, Zavacki AM, Moore DD, Edwards PA: Identification of the DNA binding specificity and potential target genes for the farnesoid X-activated receptor. J Biol Chem 2000;275:1063810647.

-31 Barbier 0, Torra IP, Sirvent A, Claudel T, Blanquart C, Duran-Sandoval D, Kuipers F, Kosykh V, Fruchart JC, Staels B: FXR induces the UGT2B4 enzyme in hepatocytes: a potential mechanism of negative feedback control of FXR activity. Gastroenterology 2003;124:1926-1940.

32 Wang H, Chen J, Hollister K, Sowers LC, Forman BM: Endogenous bile acids are ligands for the nuclear receptor FXR/BAR. Mol Cell 1999;3:543-553.

-33 Lu TT, Makishima M, Repa JJ, Schoonjans K, Kerr TA, Auwerx J, Mangelsdorf DJ: Molecular basis for feedback regulation of bile acid synthesis by nuclear receptors. Mol Cell 2000;6:507-515.

-34 Claudel T, Sturm E, Duez H, Torra IP, Sirvent A, Kosykh V, Fruchart JC, Dallongeville J, Hum DW, Kuipers F, Staels B: Bile acid-activated nuclear receptor FXR suppresses apolipoprotein A-I transcription via a negative FXR response element. J Clin Invest 2002;109:961-971.

- 35 Urizar NL, Dowhan DH, Moore DD: The farnesoid X-activated receptor mediates bile acid activation of phospholipid transfer protein gene expression. J Biol Chem 2000;275:39313-39317.

-36 Kast HR, Nguyen CM, Sinal CJ, Jones SA, Laffitte BA, Reue K, Gonzalez FJ, Willson TM, Edwards PA: Farnesoid Xactivated receptor induces apolipoprotein C-II transcription: a molecular mechanism linking plasma triglyceride levels to bile acids. Mol Endocrinol 2001;15:1720-1728.

-37 Fernández-Barrena MG, Monte MJ, Latasa MU, Uriarte I, Vicente E, Chang HC, Rodriguez-Ortigosa CM, Elferink RO, Berasain C, Marin JJ, Prieto J, Ávila MA: Lack of Abcc3 expression impairs bile-acid induced liver growth and delays hepatic regeneration after partial hepatectomy in mice. J Hepatol 2012;56:367-373.

38 Grompe M: Principles of therapeutic liver repopulation. J Inherit Metab Dis 2006;29:421-425.

-39 Laconi E, Laconi S: Principles of hepatocyte repopulation. Semin Cell Dev Biol 2002;13:433-438.

40 Harding CO, Gibson KM: Therapeutic liver repopulation for phenylketonuria. J Inherit Metab Dis 2010;33:681-687.

41 Laconi S, PillaiS, Porcu PP, Shafritz DA, Pani P, Laconi E: Massive liver replacement by transplanted hepatocytes in the absence of exogenous growth stimuli in rats treated with retrorsine. A J Pathol 2001;158:771-777.

-42 Jiang J, Salido EC, Guha C, Wang X, Moitra R, Liu L, Roy-Chowdhury J, Roy-Chowdhury N: Correction of hyperoxaluria by liver repopulation with hepatocytes in a mouse model of primary hyperoxaluria type- 1 . Transplantation 2008;85:1253-1260.

43 Weber A, Groyer-Picard MT, Franco D, Dagher I: Hepatocyte transplantation in animal models. Liver Transpl 2009;15:7-14. 